Article

\title{
Recombinant BCG Expressing the Subunit 1 of Pertussis Toxin Induces Innate Immune Memory and Confers Protection against Non-Related Pathogens
}

\author{
Alex I. Kanno ${ }^{1}$ (D), Diana Boraschi ${ }^{2}\left(\mathbb{D}\right.$, Luciana C. C. Leite ${ }^{1}$ and Dunia Rodriguez ${ }^{1, *(D)}$ \\ 1 Laboratório de Desenvolvimento de Vacinas, Instituto Butantan, São Paulo 05503-900, SP, Brazil; \\ alex.kanno@butantan.gov.br (A.I.K.); luciana.leite@butantan.gov.br (L.C.C.L.) \\ 2 Shenzhen Institute of Advanced Technology (SIAT), Chinese Academy of Sciences (CAS), \\ Shenzhen 518055, China; diana.boraschi@itb.cnr.it \\ * Correspondence: dunia.soto@butantan.gov.br
}

check for

updates

Citation: Kanno, A.I.; Boraschi, D.;

Leite, L.C.C.; Rodriguez, D.

Recombinant BCG Expressing the Subunit 1 of Pertussis Toxin Induces Innate Immune Memory and Confers Protection against Non-Related

Pathogens. Vaccines 2022, 10, 234.

https://doi.org/10.3390/

vaccines 10020234

Academic Editor: Abu

Salim Mustafa

Received: 22 December 2021

Accepted: 1 February 2022

Published: 3 February 2022

Publisher's Note: MDPI stays neutral with regard to jurisdictional claims in published maps and institutional affiliations.

Copyright: (C) 2022 by the authors. Licensee MDPI, Basel, Switzerland. This article is an open access article distributed under the terms and conditions of the Creative Commons Attribution (CC BY) license (https:// creativecommons.org/licenses/by/ $4.0 /)$.

\begin{abstract}
BCG has shown the ability to induce protection against unrelated pathogens, which likely depends on an immune mechanism known as innate immune memory or trained immunity. In this study, we evaluated the induction of innate memory by a recombinant BCG strain expressing the genetically detoxified S1 subunit of the pertussis toxin (rBCG-S1PT). In vitro pre-exposure of naïve murine macrophages to rBCG-S1PT increased their innate/inflammatory response (IL- 6 , TNF- $\alpha$, and IL-10) to a subsequent challenge with unrelated pathogens, as compared to pre-exposure to wild-type BCG. Following LPS challenge, mice immunized with rBCG-S1PT produced higher levels of IFN- $\gamma$, while the release of other inflammatory cytokines was comparable to that measured after BCG immunization. SCID mice previously immunized with rBCG-S1PT and challenged with pathogenic Candida albicans displayed a similar survival curve as BCG-immunized mice but a lower CFU burden in the kidneys, suggesting an innate memory-dependent control of C. albicans infection. This study highlights the potential of recombinant BCG to increase innate immune memory and, ultimately, nonspecific protection, more effectively than wild-type BCG. To our knowledge, this is the first report describing the potential of a recombinant BCG strain to strengthen innate immune memory responses.
\end{abstract}

Keywords: BCG; recombinant BCG; rBCG-S1PT; innate immune memory; trained immunity

\section{Introduction}

The BCG vaccine has been shown to be highly protective against the development of tuberculosis (TB) in children. This centenary vaccine is in the vaccination programs of most developing countries. Different studies have shown that it protects children against severe forms of the disease; however, protection wanes with time, and adults are less protected against pulmonary TB. Several vaccine candidates are currently under clinical trials, including "improved" BCG-based recombinant vaccines (rBCG) that rely on the expression of antigens or immunomodulatory molecules to improve BCG immunogenicity and, consequently, protection induced against Mycobacterium tuberculosis [1].

Recombinant BCG expressing the genetically detoxified S1 subunit of the pertussis toxin (rBCG-S1PT) was demonstrated to induce protection in mice against Bordetella pertussis challenge. Its pathogen-specific response was consistently demonstrated in adult and neonate mice [2-4]. The distinct immunostimulating capacity of rBCG-S1PT was evident in different experimental models. For instance, rBCG-S1PT showed an enhanced inflammatory/innate activation and a greater anti-tumor effect in a mouse model of bladder cancer-a known clinical application of wild-type BCG $[5,6]$. In an experimental model of asthma, previous immunization with rBCG-S1PT enhanced Th1 lung immunity and down-modulated the allergic response induced by ovalbumin [7]. Additionally, rBCG-S1PT has shown to induce an increased inflammatory response in human peripheral blood mononuclear cells [8]. 
The first rBCG was generated about 30 years ago (reviewed in [9]). Its use against heterologous pathogens involved the expression of pathogen-specific antigens. On the other hand, the non-specific innate immune response induced by BCG has demonstrated protection against unrelated pathogens in an immunological phenomenon known today as innate immune memory or trained immunity $[10,11]$. Through this mechanism, innate cells such as macrophages and monocytes show an increased innate/inflammatory response against unrelated stimuli after a previous exposure to BCG. This involves an epigenetic reprogramming in the promoters of several inflammatory genes and a metabolic shift towards the glycolytic pathway. Protection against a fair number of unrelated pathogens, such as Candida albicans [12], Streptococcus pneumoniae [13], and H1N1 influenza virus, has been demonstrated [14]. Although BCG can modulate the immune system towards an increased response against heterologous aggressions, caution should be taken while interpreting these data. BCG can indeed increase the responsiveness to viral infections, but this does not always translate into protection [15]. Additionally, the experimental conditions that characterize the induction of innate memory in vitro need standardization [16].

Recombinant BCG strains are expected to induce trained immunity [17] in a similar fashion as wild-type BCG. rBCG strains expressing additional microbial antigens can display improved or new features in their capacity to induce innate immune memory responses. In this work, we provide experimental evidence, for the first time, that a recombinant BCG strain can afford enhanced innate memory response and protection against unrelated pathogens.

\section{Materials and Methods}

\subsection{Microorganisms and Culture Conditions}

Wild-type M. bovis BCG Moreau and previously generated rBCG-S1PT [4] were grown in Middlebrook 7H9 (Difco, Detroit, MI, USA) supplemented with $0.5 \%$ glycerol, $0.05 \%$ Tween 80 (Sigma-Aldrich ${ }^{\circledR}$, Merck KGaA, St. Louis, MO, USA), and 10\% OADC (oleic acid-albumin-dextrose-catalase; BBL, Cockeysville, MD, USA). Cultures were maintained at $37^{\circ} \mathrm{C}$ and $5 \% \mathrm{CO}_{2}$ until reaching an OD of 0.6. Then, the cells were washed with $10 \%$ glycerol and stored at $-80{ }^{\circ} \mathrm{C}$ until use. C. albicans (ATCC 90112) was cultured in YPD (10 g/L yeast extract, $20 \mathrm{~g} / \mathrm{L}$ peptone, and $20 \mathrm{~g} / \mathrm{L}$ dextrose). Staphylococcus aureus (ATCC 25923) was cultured in LB (5 g/L yeast extract, $10 \mathrm{~g} / \mathrm{L}$ tryptone, and $10 \mathrm{~g} / \mathrm{L} \mathrm{NaCl}$ ) until reaching OD 0.6. C. albicans and S. aureus were washed with phosphate-buffered saline (PBS, $137 \mathrm{mM} \mathrm{NaCl}, 2.7 \mathrm{mM} \mathrm{KCl}, 8 \mathrm{mM} \mathrm{Na}_{2} \mathrm{HPO}_{4}$, and $1.5 \mathrm{mM} \mathrm{KH}_{2} \mathrm{PO}_{4} \mathrm{pH} 7.2$ ) and resuspended in $10 \%$ glycerol. Aliquots were stored at $-80{ }^{\circ} \mathrm{C}$ until use. Before use, each microorganism was serially diluted and plated onto the respective media plates to evaluate the number of colony-forming units (CFU).

\subsection{Mice and Ethics Statement}

Five-to-seven-week-old female C57BL/6 and NOD/SCID mice were provided by Biotério Central do Instituto Butantan and Biotério Central da Faculdade de Medicina da Universidade de São Paulo (FMUSP), respectively. All procedures were performed according to the Instituto Butantan's Ethics Committee of Animal Use (CEUA) and approved under protocol 5165040219.

\subsection{Bone Marrow Extraction and Macrophage Differentiation}

C57BL/ 6 mice were euthanized by $\mathrm{CO}_{2}$ using an appropriate chamber, and the tibia and femur were removed. Bone marrow cells were flushed with RPMI-1640 (Gibco, Life Technologies, Paisley, UK) using a syringe, and cell suspension was centrifuged for $10 \mathrm{~min}$ at $200 \times g$. The cell pellet was resuspended in RPMI- 1640 supplemented with $10 \%$ of previously inactivated fetal bovine serum (FBS; Sigma-Aldrich ${ }^{\circledR}$ ) and an antibiotic-antimycotic solution (penicillin $100 \mathrm{U} / \mathrm{mL}$, streptomycin $100 \mu \mathrm{g} / \mathrm{mL}$, and amphotericin B $0.25 \mu \mathrm{g} / \mathrm{mL}$ ) (complete medium) with the addition of 30\% L929 conditioned medium as a source of M-CSF. L929 cells were originally obtained from the American Type Culture Collection 
(ATCC, Rockville, MD, USA). The cells were distributed in 6-well plates and incubated at $37^{\circ} \mathrm{C}$ and $5 \% \mathrm{CO}_{2}$ for 6 days, changing the medium on day 4 . The plates were washed with PBS twice to remove non-adherent cells, and then adherent cells were detached using a cell scraper and ice-cold RPMI-1640. The cells were centrifuged and resuspended in complete medium. The cells were counted in a Neubauer chamber, adjusted to $10^{6}$ cells $/ \mathrm{mL}$, and distributed in 96 -well plates $(100 \mu \mathrm{L} /$ well $)$ to be used in the experiments. An aliquot was used to confirm the generation of $\mathrm{CD} 11 \mathrm{~b}^{+} \mathrm{F} 4 / 80^{+}$bone marrow-derived macrophages (BMDM), using anti-mouse CD11b conjugated with PE (BD Biosciences, San Diego, CA, USA) and anti-mouse F4/ 80 conjugated with Bv401 (BioLegend, San Diego, CA, USA).

\subsection{Peritoneal Cells Collection}

After euthanasia, $5 \mathrm{~mL}$ of ice-cold PBS was injected in the mouse peritoneal cavity to wash the cavity. Peritoneal cells were recovered by centrifuging the collected fluid for $10 \mathrm{~min}$ at $200 \times \mathrm{g}$. The cells were resuspended in complete medium and counted in a Neubauer chamber. Cell density was adjusted to $10^{6}$ cells $/ \mathrm{mL}$, and the cells were distributed in 96 -well plates $\left(100 \mu \mathrm{L} /\right.$ well). The plates were incubated at $37^{\circ} \mathrm{C}$ and $5 \% \mathrm{CO}_{2}$. After $2 \mathrm{~h}$, the plates were washed twice with PBS to remove non-adherent cells, and the remaining cells (peritoneal macrophages, PM) were incubated in complete medium. An aliquot of cells was used to confirm the macrophage phenotype, as described above.

\subsection{Priming and Challenge of Macrophages with Heterologous Pathogens}

After seeding BMDM and PM $\left(1 \times 10^{6}\right.$ cells $/ \mathrm{mL} ; 100 \mu \mathrm{L} /$ well $)$, BCG or rBCG-S1PT was used to stimulate the cells at an MOI 0.1:1 (10 ${ }^{4} \mathrm{CFU}: 10^{5}$ cells/well) for $24 \mathrm{~h}$. The $24 \mathrm{~h}$ supernatant was collected to determine the "primary cytokine response". Bacteria were removed by washing the cells with RPMI-1640, and new medium was added. The cells were left to rest in culture for 6 days, changing the medium on day 4 . On day 7 , the medium was refreshed $\left(100 \mu \mathrm{L} /\right.$ well), and the cells were challenged with C. albicans $\left(10^{6} / \mathrm{mL}\right)$, S. aureus $\left(10^{6} / \mathrm{mL}\right)$, or LPS $(10 \mathrm{ng} / \mathrm{mL})$ (from E. coli O55:B5; Sigma-Aldrich ${ }^{\circledR}$ ) for $24 \mathrm{~h}$; then, the supernatants were collected to determine the "secondary cytokine response".

\subsection{Analysis of Cell Viability}

Viability across the experiments was assessed using the FVS Viability Stain (BD Biosciences) according to the manufacturer's instructions. Data were acquired using a FACS Canto II equipment and analyzed using FlowJo Software version 10 (BD Biosciences). The gating strategy is depicted in Figure S1.

\subsection{Metabolism Analysis}

Before the secondary challenge (after 6 days of resting), the supernatants were collected, and glucose and lactate were measured using a YSI Biochemical Analyzer (YSI Life Sciences, Yellow Springs, OH, USA).

\subsection{Cytokine Production}

The supernatant of cells challenged with C. albicans, S. aureus, or LPS (after $24 \mathrm{~h}$ of stimulation) or with culture medium alone (unchallenged control) was collected and frozen. The frozen samples were thawed, centrifuged, and the supernatants were assayed for inflammation-related cytokines (IL-6, IL-10, MCP-1, IFN- $\gamma$, TNF- $\alpha$, and IL-12p70) using a Cytometric Beads Array (CBA mouse inflammatory kit, BD Biosciences), according to the manufacturer's instructions. The assay lower limits of detection were between 2.5 and $17.5 \mathrm{pg} / \mathrm{mL}$, depending on the cytokine, and the higher limit was $5000 \mathrm{pg} / \mathrm{mL}$. Data were acquired using a FACS Canto II equipment and analyzed using FCAP Array Software (BD Biosciences). Production of IL-1 $\beta$ was measured by ELISA using the DuoSet ${ }^{\circledR}$ Mouse IL-1 $\beta /$ IL-1F2 (R\&D Systems, Inc., Minneapolis, MN, USA). The supernatant of cells before the challenge (after 6 days of resting) was also assayed for these cytokines to determine the activation status of resting cells. 


\subsection{In Vivo Generation of Innate Memory in Immunocompetent Mice}

C57BL/ 6 mice were immunized subcutaneously with $10^{6}$ CFU of BCG or rBCGS1PT and 30 days later were challenged with LPS (10 ng intravenously). Whole blood was collected $4 \mathrm{~h}$ later, and cytokine concentration in the serum was measured by flow cytometry using a CBA mouse inflammatory kit as previously described.

\subsection{In Vivo Challenge with C. albicans of Immunodeficient SCID Mice}

C. albicans $\left(10^{5}\right)$ was administered through the retroorbital vein to a single C57BL/6 mouse. After 7 days, both kidneys were recovered and plated onto YPD agar plates. A single colony was cultured in liquid YPD medium until reaching OD 0.6. The cells were counted in a Neubauer chamber, and $10^{5} \mathrm{C}$. albicans cells were used to inoculate another mouse. This procedure was repeated 4 times in order to increase virulence.

Immunodeficient NOD/SCID mice were administered intravenously $10^{6}$ CFU BCG or rBCG-S1PT. After two weeks, the mice were challenged with $10^{5} \mathrm{CFU}$ of virulent C. albicans, and survival was followed for 8 weeks. Other groups of mice were euthanized 2 weeks after the C. albicans challenge, and their kidneys were collected and plated onto YPD agar in serial dilutions to evaluate CFU recovery.

\subsection{Statistical Analysis}

Comparison between groups in both in vitro and in vivo experiments was performed using a two-tailed Mann-Whitney U test. Difference in the survival curves was assessed by log-rank Mantel-Cox analysis; $p$ values $<0.05$ were considered statistically significant. Statistical analysis was performed using GraphPad Prism software version 7.04.

\section{Results}

3.1. In Vitro Model for Examining the Innate Memory Induced by rBCG-S1PT

In this study, we investigated whether rBCG-S1PT would confer innate memory on murine macrophages. The in vitro model to evaluate the generation of innate memory is depicted in Figure 1.

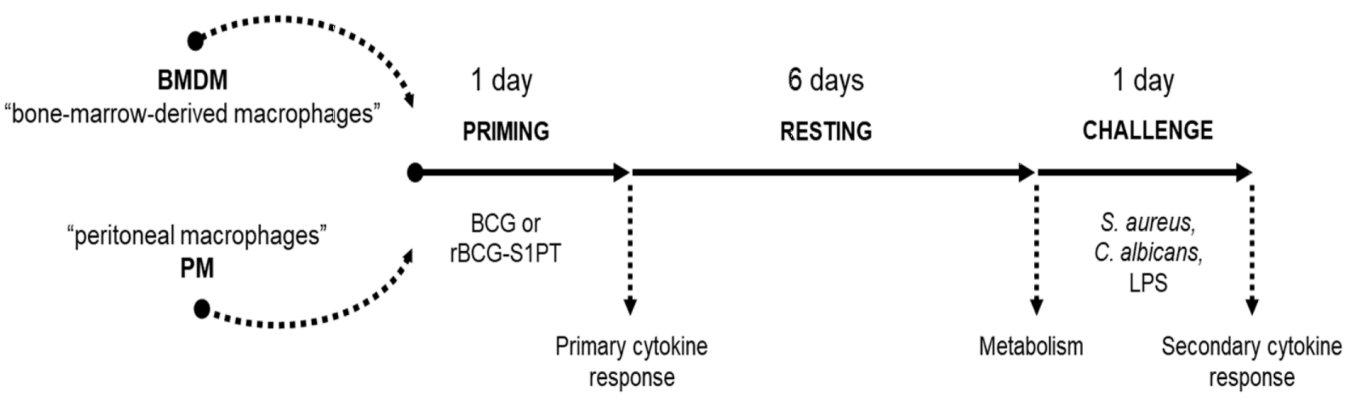

Figure 1. Schematic representation of the experimental approach using BMDM and PM. C57BL/ 6 mice were euthanized, and femur, tibia, and peritoneal cells were collected. Bone marrow cells were differentiated to macrophages (BMDM). Peritoneal macrophages (PM) were purified by adherence. BMDM and PM were primed with BCG or rBCG-S1PT for 1 day, and the supernatants were collected to determine the primary cytokine response. The cells were then left to rest for 6 days. Before the addition of the secondary stimuli (challenge), the supernatant was collected to measure glucose and lactate levels (metabolism analysis). After 1 day of re-stimulation with S. aureus, C. albicans, or LPS, the supernatants were collected to determine the secondary cytokine response.

\subsection{Primary Response of BMDM and PM to rBCG-S1PT}

We first established the rBCG dose that would confer a proper stimulation without decreasing macrophage viability. Based on our results (Table S1), we selected a MOI of 0.1:1 BCG per cell (e.g., $10^{4}$ CFU to $10^{5}$ cells), comparable to that in previous experiments using peripheral blood mononuclear cells (PBMC) [8]. 
BMDM and PM were exposed in vitro to medium alone, wild type BCG, or rBCG-S1PT. Macrophage activation (primary response) was determined $24 \mathrm{~h}$ after priming through the evaluation of IL-6, IL-10, MCP-1, IFN- $\gamma$, TNF- $\alpha$, IL-12p70, and IL-1 $\beta$. As expected, exposure of BMDM to BCG promoted the production of TNF- $\alpha$ and IL- 6 . When exposed to rBCG-S1PT, even higher levels of TNF- $\alpha$, IL-6 IL-10, MCP-1, and IL-1 $\beta$ were produced (Figure 2A). Interestingly, priming peritoneal macrophages with BCG or rBCG-S1PT induced the production of TNF- $\alpha$, IL-10, and IL-1 $\beta$, whereas the spontaneous high levels of IL-6 were not further increased by either BCG strain (Figure 2B). The cytokines IL-12p70 and IFN- $\gamma$ were detectable at very low levels and are not shown.

(A) BMDM
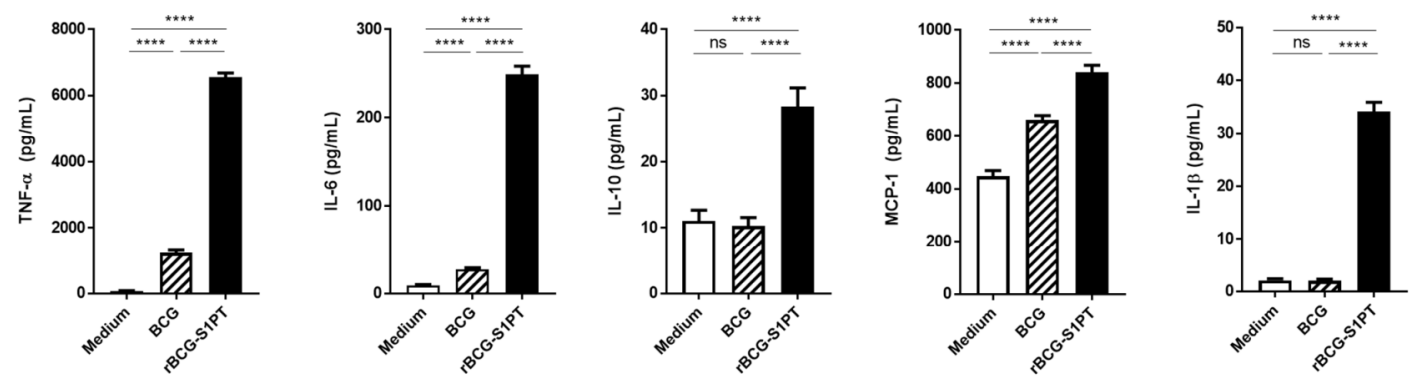

(B) Peritoneal macrophages
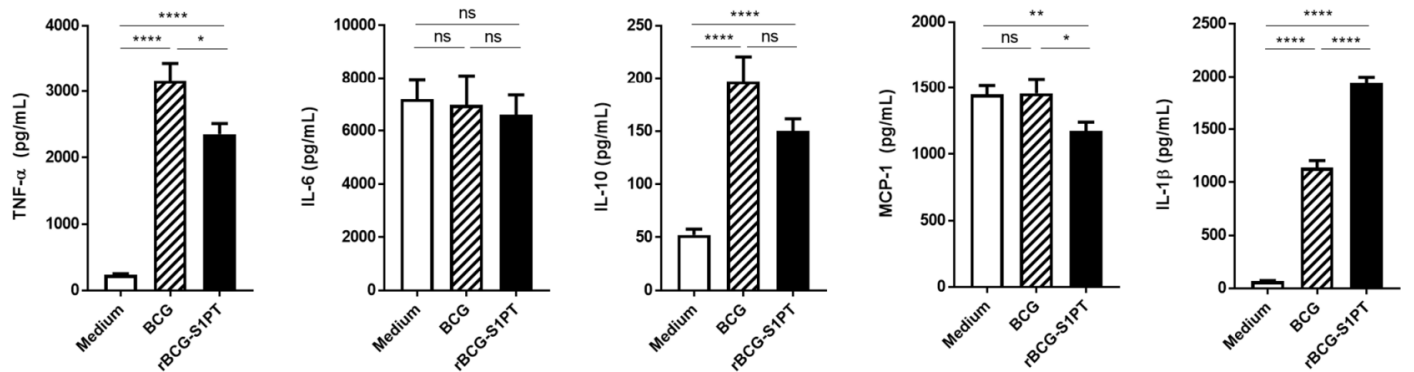

Figure 2. Primary response of BMDM (A) and peritoneal macrophages (B) to wild-type BCG or rBCG-S1PT. Bone marrow-derived macrophages and peritoneal macrophages recovered from naïve mice were exposed in vitro to culture medium alone (medium), BCG, or rBCG-S1PT (MOI 0.1:1) for $24 \mathrm{~h}$. The production of TNF- $\alpha$, IL-6, IL-10, MCP-1, and IL-1 $\beta$ (panels from the left to right) was measured in the $24 \mathrm{~h}$ supernatants. Statistical significance was assessed via Mann-Whitney U test. ${ }^{*} p<0.05,{ }^{* *} p<0.01,{ }^{* * * *} p<0.0001, \mathrm{~ns}=$ not significant. Bars represent mean $\pm \mathrm{SEM}$ of 10 replicate samples for the medium and BCG groups and of 12 replicate samples for the rBCG-S1PT group.

\subsection{Priming with rBCG-S1PT Promotes a Higher Inflammatory Response to Challenge}

Priming of BMDM and PM with BCG or rBCG-S1PT induced an increased production of inflammatory cytokines in response to the secondary heterologous challenge with C. albicans, S. aureus, or LPS (Figure 3). When assessing TNF- $\alpha$, one of the main innate/inflammatory cytokines (upper panels), priming with BCG (striped bars) increased its production by both macrophage types only in response to $C$. albicans challenge. Conversely, rBCG-S1PT priming (black bars) induced a higher TNF- $\alpha$ production upon challenge with all agents (except in PM challenged with $S$. aureus) in comparison to both unprimed cells (white bars) and cells primed with BCG. When examining the production of another inflammatory cytokine, IL-6 (middle panels), cells primed with rBCG-S1PT afforded an increase in the response upon all heterologous challenges in comparison to medium and wild-type BCG (except BMDM challenged with S. aureus). BCG-primed peritoneal macrophages also exhibited an increased secondary IL-6 response in comparison to unprimed cells (when challenged with $S$. aureus and LPS), but at lower levels. The production of the anti-inflammatory cytokine IL-10 showed a dichotomy between BMDM and PM, with rBCG-S1PT priming essentially unable to induce IL-10 in BMDM, but increasing its pro- 
duction in PM challenged with S. aureus and C. albicans. The concomitant upregulation of inflammatory and anti-inflammatory mechanisms observed here suggests that the induction of higher inflammatory responses induced by rBCG-S1PT may be accompanied by regulatory mechanisms that prevent excessive/damaging inflammatory responses.

(A) BMDM
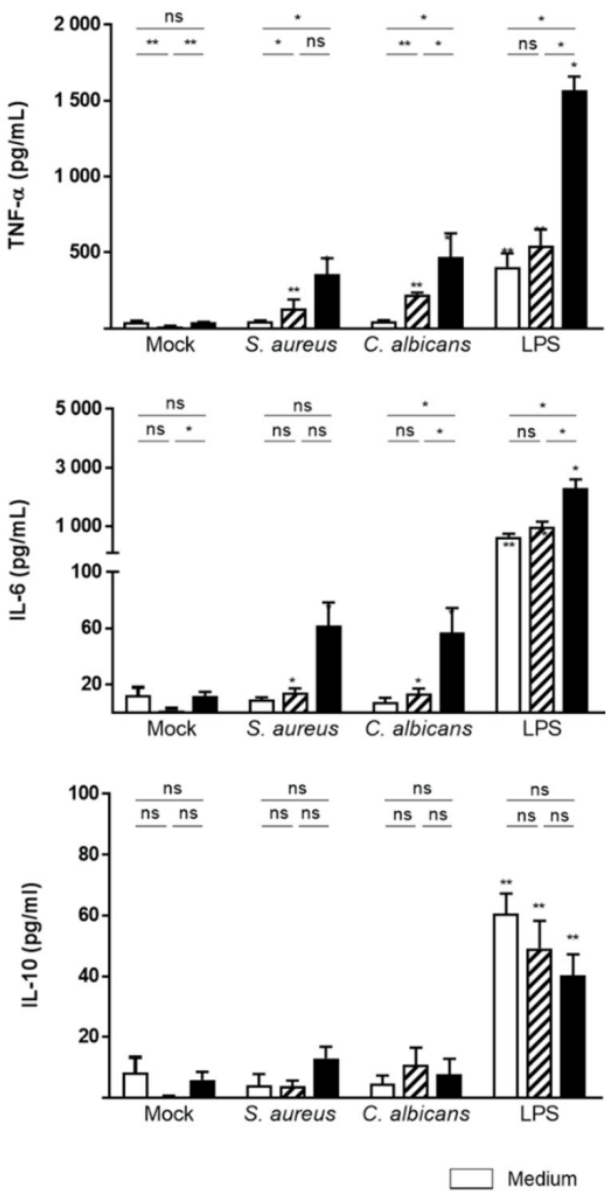

(B) Peritoneal macrophages
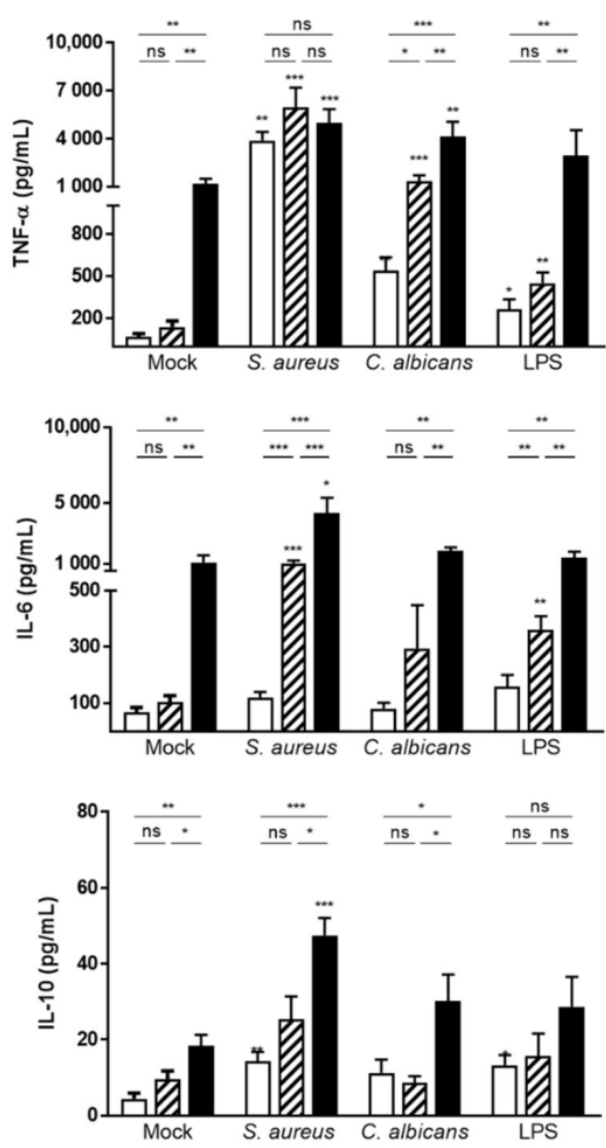

WII BCG

rBCG-S1PT

Figure 3. Memory response of primed macrophages to heterologous challenges. BMDM (A) and peritoneal macrophages (B) from naïve mice were exposed in vitro to culture medium alone (unprimed, white bars), BCG (striped bars), or rBCG-S1PT (black bars) (MOI 0.1:1) for $24 \mathrm{~h}$. Cells were then left to rest for 6 days and re-stimulated with medium alone (mock) or with S. aureus, C. albicans, or LPS (horizontal axis). The production of TNF- $\alpha$ (upper panels), IL-6 (middle panels), and IL-10 (lower panels) was measured after $24 \mathrm{~h}$. Statistical analysis was performed via Mann-Whitney U test. ${ }^{*} p<0.05,{ }^{* *} p<0.01,{ }^{* * *} p<0.001, \mathrm{~ns}=$ not significant. Bars represent mean $\pm \mathrm{SEM}$ of $4-5$ (for BMDM) and 5-8 (for peritoneal macrophages) replicate samples. Asterisks over the columns in S. aureus, C. albicans, and LPS refer to the comparison with the respective mock.

It should be noted that priming with rBCG-S1PT induced a macrophage activation that was not fully extinguished after one week (in terms of TNF- $\alpha$ and IL- 6 production in the last 2 days of resting) (Figure S2). Challenging these cells resulted in an additional increase in cytokine production (Figure 3). Notably, in our experimental conditions, the two different macrophage types exhibited distinct patterns of general cytokine response. While BMDM appeared to be more sensitive to LPS, peritoneal macrophages reacted better to $S$. aureus and C. albicans (see white columns between mock and challenges in Figure 3 and note the different values on the vertical axis between BMDM and PM). The cytokines IL-12p70 and IFN- $\gamma$ were detectable at very low levels and are not shown. Data for MCP-1 and IL-1 $\beta$ are shown in Figure S3. While no significant memory effect was detectable for 
IL-1 $\beta$ production, it is notable that the production of MCP-1 in BMDM (but not in PM) in response to LPS was significantly lower in BCG- and rBCG-primed cells, in a typical tolerance type of memory.

\section{4. rBCG-S1PT Induces Metabolic Changes in Memory Macrophages}

Previous studies reported a shift towards the glycolytic pathway in memory macrophages. Here, priming with rBCG-S1PT promoted a higher consumption of glucose, linked with the production of lactate in macrophages left to rest for 6 days after priming. In BMDM, a significant shift in the lactate/glucose ratio was observed (Figure 4A). In peritoneal macrophages, rBCG-S1PT did not induce a greater consumption of glucose in memory cells, yet a higher lactate concentration was observed, with a consequent significant shift of the lactate/glucose ratio (Figure 4B).

\section{(A) BMDM}
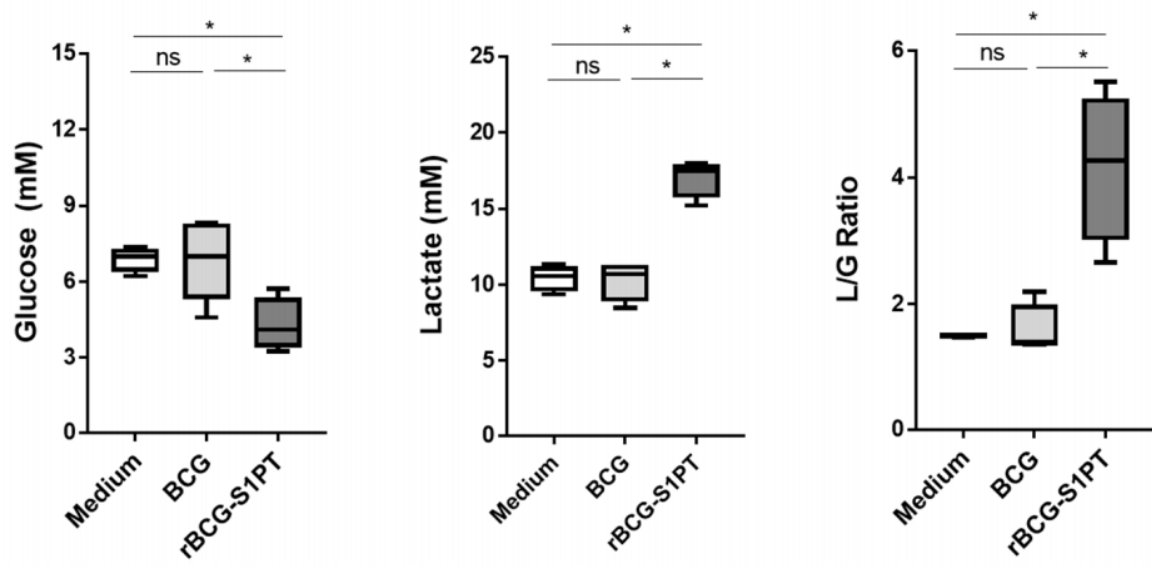

(B) Peritoneal macrophages
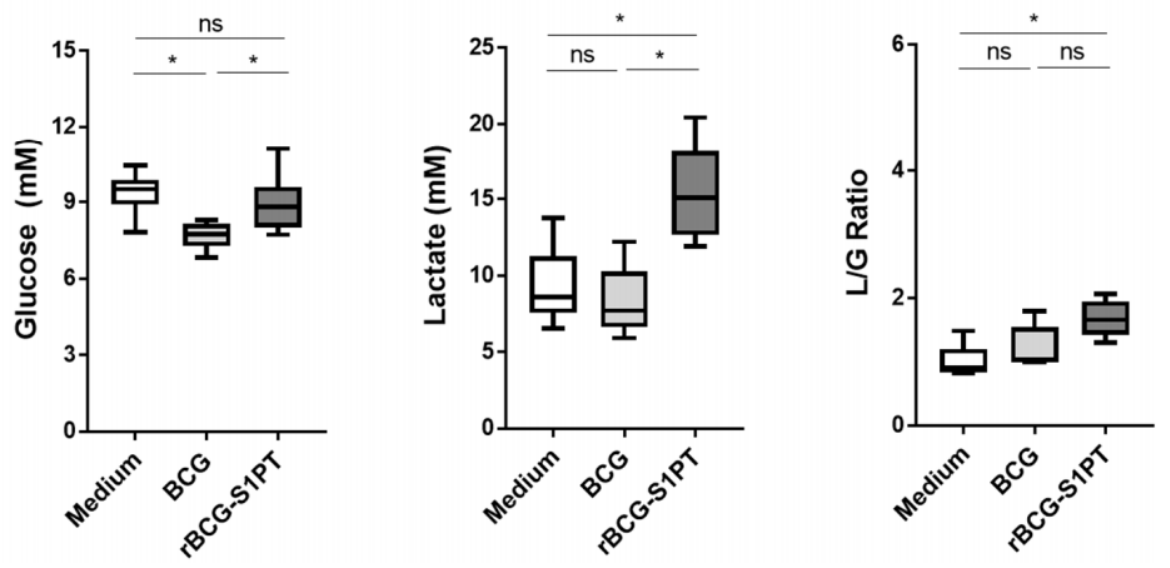

Figure 4. rBCG-S1PT induces metabolic changes in memory macrophages. BMDM (A) and PM (B) from naïve mice were exposed in vitro to culture medium alone (medium), BCG, or rBCGS1PT (MOI 0.1:1), then left to rest in fresh medium for 6 days. Glucose and lactate levels and the lactate/glucose $(\mathrm{L} / \mathrm{G})$ ratio were compared between groups. Statistical analysis was performed via Mann-Whitney U test. ${ }^{*} p<0.05$, ns = not significant. Boxes and whiskers represent value distribution with median (horizontal line), first, and third quartile (box limits) and minimum and maximum values (whiskers) of four replicate samples for BMDM and six replicate samples for PM.

\section{5. rBCG-S1PT Induces an Increased Cytokine Response upon LPS Challenge In Vivo}

To test whether rBCG-S1PT could enhance the response to a heterologous challenge in vivo, C57BL/ 6 mice were subcutaneously administered saline (control), BCG, or rBCGS1PT and challenged intravenously with LPS 4 weeks later. The cytokine levels in the 
serum were measured before and $4 \mathrm{~h}$ after the challenge. Mice primed with BCG displayed an increased production of TNF- $\alpha$ upon LPS stimulation, while the production of other cytokines was comparable to that of controls (Figure 5). In rBCG-S1PT-primed mice, a priming-dependent increase was evident for TNF- $\alpha$, IL- $1 \beta$, and IFN- $\gamma$. Notably, rBCGS1PT-primed mice reacted to LPS with an increase in the circulating levels of IFN- $\gamma$ that was significantly higher than that observed in BCG-primed mice.
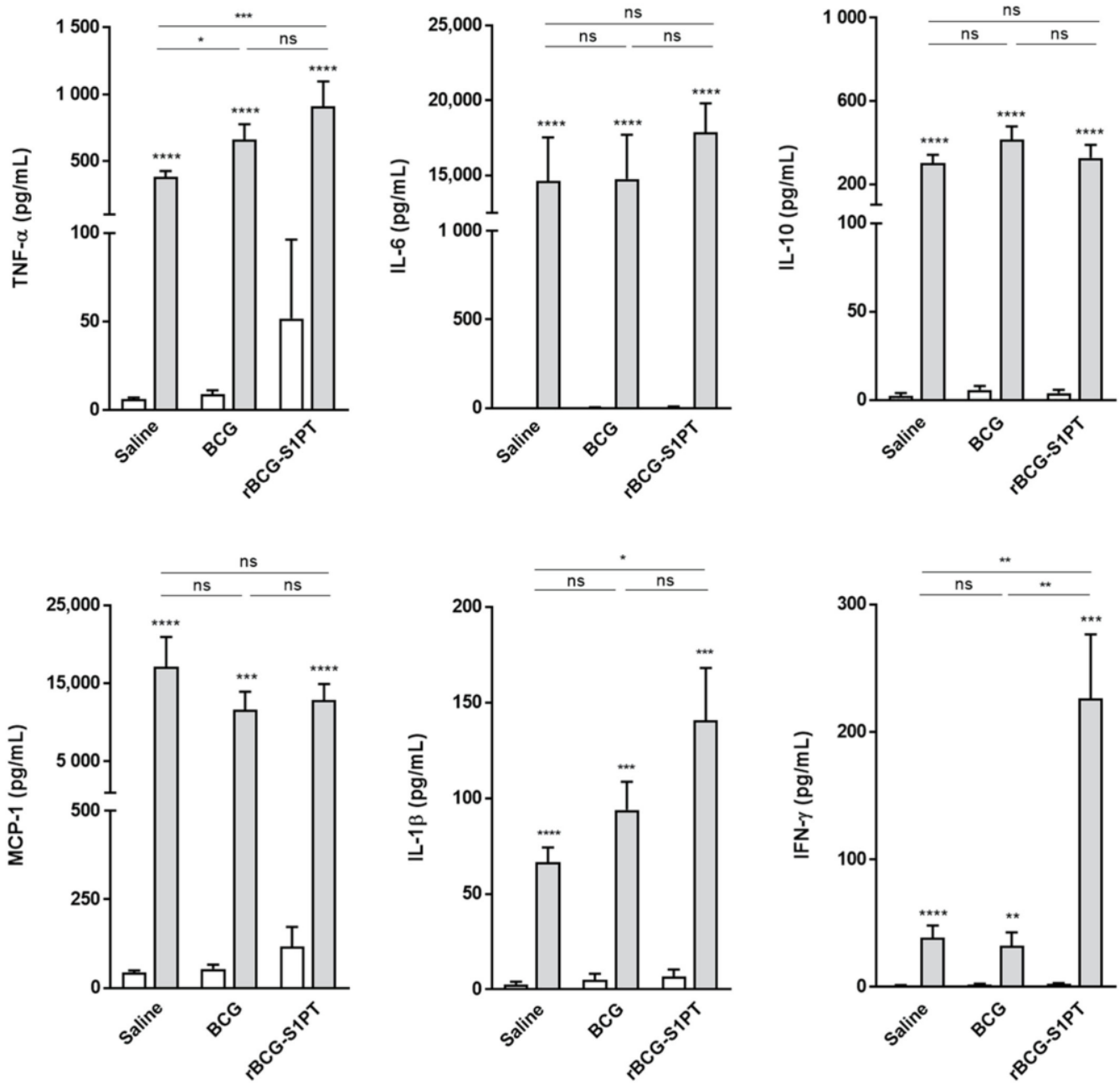

Figure 5. Priming of mice with rBCG-S1PT enhances the cytokine response to LPS challenge. Mice were administered saline or $10^{6}$ CFU of BCG or rBCG-S1PT and challenged with LPS 4 weeks later. The cytokine levels in the serum were determined before (white bars) and $4 \mathrm{~h}$ after the challenge (gray bars). Statistical analysis was performed via Mann-Whitney U test. ${ }^{*} p<0.05,{ }^{* *} p<0.01,{ }^{* * *} p<0.001$, **** $p<0.0001, \mathrm{~ns}=$ not significant. Bars represent mean \pm SEM of values from 10 mice. Asterisks over gray columns represent the comparison with the respective control group (white column).

\subsection{Previous Immunization with rBCG-S1PT Increases Protection against C. albicans Challenge}

BCG has been shown to induce non-specific protection against heterologous pathogens. Here, we evaluated whether rBCG-S1PT could afford this non-specific protection in vivo. SCID mice were administered saline, BCG, or rBCG-S1PT and challenged 2 weeks later with C. albicans. Survival was followed for 8 weeks after the challenge. Both BCG and rBCG-S1PT improved mice survival in comparison to the saline group (Figure 6A). When mice were evaluated for $\mathrm{CFU}$ in the kidneys one week after the challenge, a significant reduction in CFU was observed in the group primed with rBCG-S1PT (mean $51.5 \times 10^{3} \mathrm{CFU} /$ kidney) in comparison to that treated with saline (mean $260.2 \times 10^{3} \mathrm{CFU} /$ kidney), whereas the effect of BCG was not significant (mean $203.6 \times 10^{3}$ CFU/kidney) (Figure 6B). 
(A)

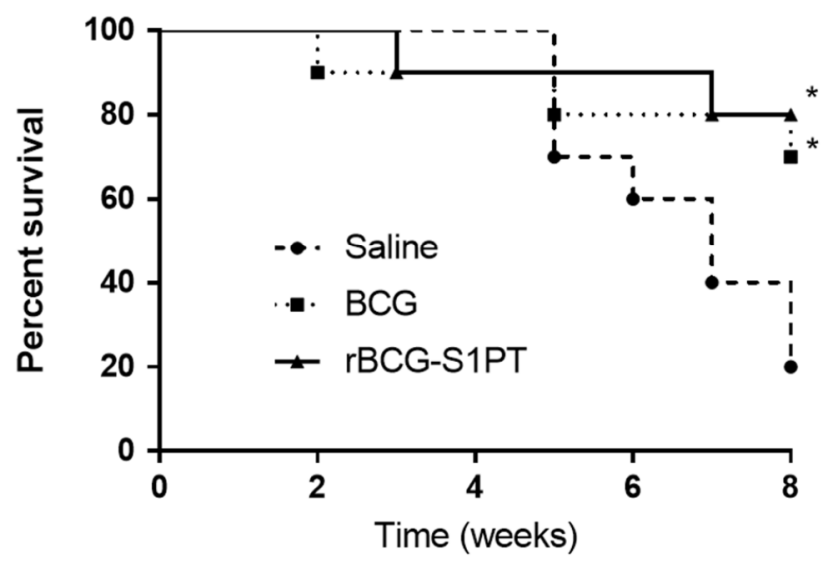

(B)

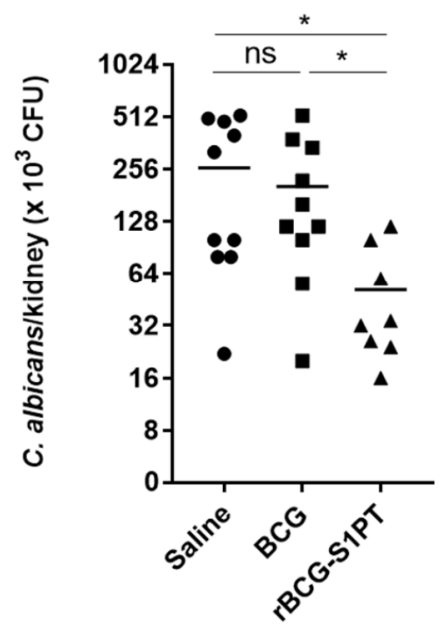

Figure 6. Priming with rBCG-S1PT increases the protection against $C$. albicans challenge. (A) Groups of SCID mice ( $n=10$ /group) were primed with $10^{6}$ CFU of BCG or rBCG-S1PT and challenged 2 weeks later with $C$. albicans. The mice were followed for survival for 8 weeks after infection. (B) Using other groups of mice ( $n=5$ /group), kidneys were collected one week after the challenge, and $C$. albicans CFU was evaluated. The results are representative of two and three experiments, respectively. The statistical significance of survival was determined via Log-rank (Mantel-Cox) test, and CFU via Mann-Whitney U test. ${ }^{*} p<0.05$ vs. saline control. $\mathrm{ns}=$ not significant. Bars represent the mean \pm SEM of values from the kidneys of five mice.

\section{Discussion}

In this study, we demonstrate that a recombinant BCG vaccine, rBCG-S1PT, is able to induce an innate immune memory response. To date, few agents have been shown to induce innate immune memory, and each of them seems to induce a different profile. BCG, LPS, and $\beta$-glucan are often used in experimental conditions [12,18-20], but only BCG is used as a human vaccine and has shown beneficial nonspecific effects in clinical [21] and epidemiological settings [22]. BCG encompasses a variety of molecules that may trigger innate immunity through several pathways. For instance, many components of the mycobacterial cell wall such as peptidoglycan, arabinogalactan, and mycolic acids are sensed by TLR2 and TLR4 [23]. Specific proteins such as Ag85 can directly act as a TLR agonist [24]. Additionally, as a live attenuated vaccine, BCG viability is a factor that may also affect its protective efficacy [25].

The intrinsic adjuvanticity of BCG, i.e., its capacity to non-specifically amplify the immune response, is one of the reasons supporting the generation of hundreds of recombinant strains in the last 30 years [9]. rBCG-S1PT was initially devised as a neonatal vaccine against pertussis [2]. It protects adult and neonate mice against $B$. pertussis challenge, exhibiting an increased Th1-predominant immune response in this animal model. When repurposed to immunotherapy in a mouse bladder cancer orthotopic model, rBCG-S1PT showed a greater reduction of the bladder's tumor weight and an increased survival time [5]. The anti-tumor effect was related to a higher expression of TNF- $\alpha$ and IL-10 in the bladder [6]. In the present study, BMDM stimulated with rBCG-S1PT showed a marked increase in the production of IL-6, TNF- $\alpha$, IL-10 MCP-1, and IL- $1 \beta$ in comparison to cells stimulated with BCG. Conversely, the primary response induced by rBCG-S1PT in peritoneal macrophages showed increased IL-1 $\beta$ but lower TNF- $\alpha$ and IL-10 production in comparison to that induced by wild-type BCG. In previous studies, our group showed that human PBMC stimulated with rBCG-S1PT produce increased levels of IL-8, IL-6, and IL-10, but similar levels of IL- $1 \beta$ and TNF- $\alpha$ in comparison to cells stimulated with wild-type BCG [8]. This highlights that immune activation induced by rBCG-S1PT is different in distinct cell populations. 
In our experimental conditions, BMDM and peritoneal macrophages exposed to rBCG-S1PT maintained a significant cytokine production even after one week from the initial stimulation, differently from the extinction of the primary response in macrophages exposed to wild-type BCG. This suggests that rBCG-S1PT can promote a higher and more prolonged inflammatory response.

Re-stimulation of rBCG-S1PT-primed macrophages with S. aureus, C. albicans, and LPS further increased their response, in terms of TNF- $\alpha$ and IL-6 production. In BMDM, both wild-type BCG and rBCG-S1PT appeared to induce the classical innate memory "recall" response (primary response, extinction of activation, memory response), and the recombinant strain generated a sustained unique response. Conversely, the type of response observed in peritoneal macrophages may be considered a non-classical memory response, in which primed cells acquire a long-lasting activation status that can be further upregulated upon subsequent challenges. This type of innate memory is well known in invertebrates with the name of "sustained unique response" or "acquired resistance" [26-30]. Thus, rBCGS1PT, and likely other rBCG strains, may differ from wild-type BCG in the type of innate memory and cell reprogramming they generate. Additionally, the cytokine response of peritoneal macrophages was overall higher than in BMDM. This is an important observation, as it shows that different macrophage populations (as for instance macrophages residing in different organs) can develop different innate memory profiles to the same combination of priming/challenge agents. This observation underlines the importance of a local, organ-targeted induction of innate memory in future preventive or therapeutic interventions.

While innate memory generation was evident in macrophages after heterologous challenge, the Warburg effect (enhanced aerobic glycolysis with lactate production, generally associated with innate memory generated by $\beta$-glucan) was not clearly observed. This may depend on the fact that memory induced by BCG is not necessarily associated with the Warburg effect, since BCG can concomitantly increase both glycolysis and oxidative phosphorylation [31]. Notably, however, the Warburg effect was evident in rBCG-S1PTprimed BMDM, while it was absent in BCG-primed cells. This observation confirms the dissociation between Warburg effect and innate memory induced by BCG (no Warburg effect in BCG-primed cells).

We sought to investigate if administration of rBCG-S1PT would induce innate immune memory responses in vivo. Mice immunized with BCG or rBCG-S1PT produced higher and similar amounts of TNF- $\alpha$ in the serum upon LPS challenge. Interestingly, only rBCG-S1PT was able to induce significant levels of IL- $1 \beta$ and IFN- $\gamma$, which are inflammatory cytokines important for the establishment of innate memory in humans and mice, respectively [32,33]. The increased production of IFN- $\gamma$ confirms previous data reporting a Th1-biased effect of rBCG-S1PT in comparison to wild-type BCG [2]. It is interesting to note that IFN- $\gamma$ was only induced in rBCG-S1PT-primed mice upon re-stimulation with LPS, while negligible levels were detected before the challenge.

BCG immunization was shown to protect mice against $C$. albicans infection, assessed both as CFU burden and as survival [12]. Our results confirm the non-specific protection induced by BCG against C. albicans. To better understand the role of rBCG-S1PT in inducing innate immune memory, we administered BCG or rBCG-S1PT to SCID mice, which lack T and B cell responses, and challenged them with $C$. albicans 2 weeks later. Immunization with rBCG-S1PT caused a mean reduction of $80 \%$ in the C. albicans burden in the kidneys compared to untreated mice, while wild-type BCG only caused a non-significant reduction of $20 \%$. Previous studies reported a 10 -fold reduction in C. albicans CFU in the kidneys of SCID mice immunized with BCG [12]. These differences may be due the distinct BCG strain (Danish versus Moreau) or the dose used $\left(750 \mu \mathrm{g} /\right.$ mice versus $\left.10^{6} \mathrm{CFU} / \mathrm{mice}\right)$. Overall, however, no significant difference in survival was observed between mice treated with rBCG-S1PT and those treated with the parental BCG strain, of which both reduced mortality to a similar extent. The observation of BCG-induced non-specific protection in experimental models has been variable, also in the case of epidemiological data [22]. For instance, BCGinduced innate immune memory can increase efferocytosis of alveolar phagocytes against 
H1N1 [34], but this does not translate into protection of mice against H7N9 challenge [15]. Furthermore, as already mentioned, the innate memory effects are likely different among the organs, and their generation in specific locations may strongly depend on the route of administration and the dosage of the memory-inducing agent $[15,35]$. How innate memory is generated is an issue that is being actively discussed and investigated $[10,36]$.

Recombinant BCG strains can maintain the immunostimulatory characteristics of wildtype BCG on the innate immune response, while able to induce adaptive immunity against $\mathrm{BCG}$ and recombinant antigens. It is therefore expected that $\mathrm{rBCG}$ vaccines could stimulate both protective innate and adaptive immune responses [17]. To date, no rBCG vaccine has been experimentally evaluated for the induction of non-specific immune memory. The closest vaccine evaluated was the $M$. tuberculosis-based attenuated vaccine (MTBVAC), an improved TB vaccine candidate. MTBVAC is in phase II clinical trial and demonstrated protection of mice [37] and rhesus macaques against $M$. tuberculosis aerosol challenge [38]. Previous immunization with MTBVAC can also enhance the immune response in synergy with the pertussis vaccine [39], while having the same capacity as BCG to induce innate memory [40].

The BCG vaccine has been used for a century, and even today new features are still being discovered. The suggestion that innate immune memory could explain why BCG can offer protection against unrelated diseases brought a renewed attention to this vaccine. Moreover, BCG is being repurposed to treat other diseases, and studies investigating its effect against SARS-CoV-2 are under evaluation [41-43]. The expression of heterologous antigens in BCG should maintain the original characteristic and can introduce new ones, as demonstrated in this work with rBCG-S1PT. Here, we provide experimental evidence that rBCG-S1PT is able to increase innate immune memory and, ultimately, non-specific protection.

\section{Conclusions}

In this work, we demonstrated that rBCG-S1PT can enhance the innate immune memory response and induce an increased non-specific protection against C. albicans challenge. Further studies to phenotype the involved macrophage population and/or address the role of other innate cells can provide additional evidence on the mechanisms involved. To our knowledge, this is the first report describing the capacity of a recombinant BCG strain to afford improved innate immune memory responses.

Supplementary Materials: The following supporting information can be downloaded at: https: //www.mdpi.com/article/10.3390/vaccines10020234/s1. Figure S1: Gating strategy to identify macrophages and their viability. Cell suspensions from the peritoneal cavity and differentiated bone marrow cells were gated for single cells and then live/dead cells according to a negative selection gate based on dead cells (with methanol). Live cells double positive for CD11b ${ }^{+}$and F4/80 ${ }^{+}$were considered as live macrophages. Table S1: Macrophage viability in culture following exposure to BCG and rBCG-S1PT at different MOIs. Figure S2: Activation state of primed BMDM and peritoneal macrophages after the 6-day resting phase. Macrophages were exposed for $24 \mathrm{~h}$ to culture medium, alone (medium) or containing BCG or rBCG-S1PT at MOI 0.1:1. The cells were then washed and incubated in fresh medium for 6 days, changing the medium on day 4 . The supernatants were collected on day 6, and the cytokines produced in the days 5-6 were measured. Statistical analysis was performed via Mann-Whitney U test. ${ }^{*} p<0.05,{ }^{* *} p<0.01,{ }^{* * *} p<0.001$, ns $=$ not significant. Bars represent mean \pm SEM of 5-7 replicate samples. Figure S3: Memory response of primed macrophages to heterologous challenges. BMDM (A) and peritoneal macrophages (B) from naïve mice were exposed in vitro to culture medium alone (unprimed, white bars), BCG (striped bars), or rBCG-S1PT (black bars) (MOI 0.1:1) for $24 \mathrm{~h}$. The cells were then left to rest for 6 days and re-stimulated with medium alone (mock) or with S. aureus, C. albicans, or LPS (horizontal axis). The production of MCP-1 (upper panels) and IL-1 $\beta$ (lower panels) was measured after $24 \mathrm{~h}$. Statistical analysis was carried out via Mann-Whitney U test. ${ }^{*} p<0.05,{ }^{* *} p<0.01,{ }^{* * *} p<0.001$, ns $=$ not significant. Asterisks over the columns in S. aureus, C. albicans, and LPS represent the comparison with the respective mock. Bars represent mean \pm SEM of 6-7 replicate samples. 


\begin{abstract}
Author Contributions: Conceptualization, A.I.K., L.C.C.L., D.B. and D.R.; methodology, A.I.K. and D.R.; formal analysis, A.I.K. and D.R.; data curation, A.I.K., L.C.C.L., D.B. and D.R.; writingoriginal draft preparation, A.I.K., L.C.C.L. and D.R.; writing-review and editing, A.I.K., D.B., L.C.C.L. and D.R.; project administration, L.C.C.L. and D.R.; funding acquisition, L.C.C.L. All authors have read and agreed to the published version of the manuscript.
\end{abstract}

Funding: This research was funded by FUNDAÇÃO DE AMPARO À PESQUISA DO ESTADO DE SÃO PAULO (FAPESP), grant number 2017/24832-6.

Institutional Review Board Statement: The study was conducted according to the guidelines of the Declaration of Helsinki and approved by the Ethics Committee on Animal Use of Butantan Institute (protocol 5165040219 approval 20 March 2019).

Informed Consent Statement: Not applicable.

Data Availability Statement: The data presented in this study are available on request from the corresponding author.

Acknowledgments: We sincerely thank Márcia Regina Franzolin of the Laboratório de Bacteriologia for the kind donation of the C. albicans and S. aureus strains. Renato Mancini Astray and Jaci Leme for the technical assistance in the use of the YSI Biochemical Analyzer. Momtchilo Russo and Eliane Gomes from Universidade de São Paulo for the kind donation of the L929 cell line.

Conflicts of Interest: Luciana C.C. Leite has a patent application on the use of rBCG-S1PT in bladder cancer treatment. The funders had no role in the design of the study; in the collection, analyses, or interpretation of data; in the writing of the manuscript, or in the decision to publish the results.

\title{
References
}

1. $\quad$ Lange, C.; Aaby, P.; Behr, M.A.; Donald, P.R.; Kaufmann, S.H.E.; Netea, M.G.; Mandalakas, A.M. 100 years of Mycobacterium bovis bacille Calmette-Guerin. Lancet Infect. Dis. 2021, 22, e2-e12. [CrossRef]

2. Nascimento, I.P.; Dias, W.O.; Mazzantini, R.P.; Miyaji, E.N.; Gamberini, M.; Quintilio, W.; Gebara, V.C.; Cardoso, D.F.; Ho, P.L.; Raw, I.; et al. Recombinant Mycobacterium bovis BCG expressing pertussis toxin subunit S1 induces protection against an intracerebral challenge with live Bordetella pertussis in mice. Infect. Immun. 2000, 68, 4877-4883. [CrossRef] [PubMed]

3. Nascimento, I.P.; Dias, W.O.; Quintilio, W.; Christ, A.P.; Moraes, J.F.; Vancetto, M.D.; Ribeiro-Dos-Santos, G.; Raw, I.; Leite, L.C. Neonatal immunization with a single dose of recombinant BCG expressing subunit $\mathrm{S} 1$ from pertussis toxin induces complete protection against Bordetella pertussis intracerebral challenge. Microbes Infect. 2008, 10, 198-202. [CrossRef]

4. Nascimento, I.P.; Dias, W.O.; Quintilio, W.; Hsu, T.; Jacobs, W.R., Jr.; Leite, L.C. Construction of an unmarked recombinant BCG expressing a pertussis antigen by auxotrophic complementation: Protection against Bordetella pertussis challenge in neonates. Vaccine 2009, 27, 7346-7351. [CrossRef] [PubMed]

5. Andrade, P.M.; Chade, D.C.; Borra, R.C.; Nascimento, I.P.; Villanova, F.E.; Leite, L.C.; Andrade, E.; Srougi, M. The therapeutic potential of recombinant BCG expressing the antigen S1PT in the intravesical treatment of bladder cancer. Urol. Oncol. Semin. Orig. Investig. 2010, 28, 520-525. [CrossRef]

6. Chade, D.C.; Borra, R.C.; Nascimento, I.P.; Villanova, F.E.; Leite, L.C.; Andrade, E.; Srougi, M.; Ramos, K.L.; Andrade, P.M. Immunomodulatory effects of recombinant BCG expressing pertussis toxin on TNF-alpha and IL-10 in a bladder cancer model. J. Exp. Clin. Cancer Res. 2008, 27, 78. [CrossRef]

7. Christ, A.P.; Rodriguez, D.; Bortolatto, J.; Borducchi, E.; Keller, A.; Mucida, D.; Silva, J.S.; Leite, L.C.; Russo, M. Enhancement of Th1 lung immunity induced by recombinant Mycobacterium bovis Bacillus Calmette-Guerin attenuates airway allergic disease. Am. J. Respir. Cell Mol. Biol. 2010, 43, 243-252. [CrossRef]

8. Rodriguez, D.; Goulart, C.; Pagliarone, A.C.; Silva, E.P.; Cunegundes, P.S.; Nascimento, I.P.; Borra, R.C.; Dias, W.O.; Tagliabue, A.; Boraschi, D.; et al. In vitro Evidence of Human Immune Responsiveness Shows the Improved Potential of a Recombinant BCG Strain for Bladder Cancer Treatment. Front. Immunol. 2019, 10, 1460. [CrossRef]

9. Marques-Neto, L.M.; Piwowarska, Z.; Kanno, A.I.; Moraes, L.; Trentini, M.M.; Rodriguez, D.; Silva, J.; Leite, L.C.C. Thirty years of recombinant BCG: New trends for a centenary vaccine. Expert Rev. Vaccines 2021, 20, 1001-1011. [CrossRef]

10. Boraschi, D.; Italiani, P. Innate Immune Memory: Time for Adopting a Correct Terminology. Front. Immunol. 2018, 9, 799. [CrossRef]

11. Netea, M.G.; Quintin, J.; van der Meer, J.W. Trained immunity: A memory for innate host defense. Cell Host Microbe 2011, 9, 355-361. [CrossRef] [PubMed]

12. Kleinnijenhuis, J.; Quintin, J.; Preijers, F.; Joosten, L.A.; Ifrim, D.C.; Saeed, S.; Jacobs, C.; van Loenhout, J.; de Jong, D.; Stunnenberg, H.G.; et al. Bacille Calmette-Guerin induces NOD2-dependent nonspecific protection from reinfection via epigenetic reprogramming of monocytes. Proc. Natl. Acad. Sci. USA 2012, 109, 17537-17542. [CrossRef] [PubMed] 
13. Tarancon, R.; Dominguez-Andres, J.; Uranga, S.; Ferreira, A.V.; Groh, L.A.; Domenech, M.; Gonzalez-Camacho, F.; Riksen, N.P.; Aguilo, N.; Yuste, J.; et al. New live attenuated tuberculosis vaccine MTBVAC induces trained immunity and confers protection against experimental lethal pneumonia. PLoS Pathog. 2020, 16, e1008404. [CrossRef] [PubMed]

14. Spencer, J.C.; Ganguly, R.; Waldman, R.H. Nonspecific protection of mice against influenza virus infection by local or systemic immunization with Bacille Calmette-Guerin. J. Infect. Dis. 1977, 136, 171-175. [CrossRef]

15. De Bree, L.C.J.; Marijnissen, R.J.; Kel, J.M.; Rosendahl Huber, S.K.; Aaby, P.; Benn, C.S.; Wijnands, M.V.W.; Diavatopoulos, D.A.; van Crevel, R.; Joosten, L.A.B.; et al. Bacillus Calmette-Guerin-Induced Trained Immunity Is Not Protective for Experimental Influenza A/Anhui/1/2013 (H7N9) Infection in Mice. Front. Immunol. 2018, 9, 869. [CrossRef]

16. Bekkering, S.; Blok, B.A.; Joosten, L.A.; Riksen, N.P.; van Crevel, R.; Netea, M.G. In Vitro Experimental Model of Trained Innate Immunity in Human Primary Monocytes. Clin. Vaccine Immunol. 2016, 23, 926-933. [CrossRef]

17. Covian, C.; Fernandez-Fierro, A.; Retamal-Diaz, A.; Diaz, F.E.; Vasquez, A.E.; Lay, M.K.; Riedel, C.A.; Gonzalez, P.A.; Bueno, S.M.; Kalergis, A.M. BCG-Induced Cross-Protection and Development of Trained Immunity: Implication for Vaccine Design. Front. Immunol. 2019, 10, 2806. [CrossRef]

18. Dos Santos, J.C.; Barroso de Figueiredo, A.M.; Teodoro Silva, M.V.; Cirovic, B.; de Bree, L.C.J.; Damen, M.; Moorlag, S.; Gomes, R.S.; Helsen, M.M.; Oosting, M.; et al. beta-Glucan-Induced Trained Immunity Protects against Leishmania braziliensis Infection: A Crucial Role for IL-32. Cell Rep. 2019, 28, 2659-2672.e6. [CrossRef]

19. Italiani, P.; Boraschi, D. Induction of Innate Immune Memory by Engineered Nanoparticles: A Hypothesis that May Become True. Front. Immunol. 2017, 8, 734. [CrossRef]

20. Seeley, J.J.; Ghosh, S. Molecular mechanisms of innate memory and tolerance to LPS. J. Leukoc. Biol. 2017, 101, 107-119. [CrossRef]

21. Morales, A.; Eidinger, D.; Bruce, A.W. Intracavitary Bacillus Calmette-Guerin in the treatment of superficial bladder tumors. J. Urol. 1976, 116, 180-183. [CrossRef]

22. WHO. SAGE Meeting of April 2014. Session: Non-Specific Effects of Vaccines on Mortality in Children under 5 Years of Age. 2014. Available online: https://www.who.int/immunization/sage/meetings/2014/april/presentations_background_docs/ en/index1.html (accessed on 14 January 2021).

23. Basu, J.; Shin, D.M.; Jo, E.K. Mycobacterial signaling through toll-like receptors. Front. Cell Infect. Microbiol. $2012,2,145$. [CrossRef] [PubMed]

24. Dockrell, H.M.; Smith, S.G. What Have We Learnt about BCG Vaccination in the Last 20 Years? Front. Immunol. 2017, 8, 1134. [CrossRef] [PubMed]

25. Bickett, T.E.; McLean, J.; Creissen, E.; Izzo, L.; Hagan, C.; Izzo, A.J.; Silva Angulo, F.; Izzo, A.A. Characterizing the BCG Induced Macrophage and Neutrophil Mechanisms for Defense Against Mycobacterium tuberculosis. Front. Immunol. 2020, $11,1202$. [CrossRef]

26. Cooper, D.; Eleftherianos, I. Memory and Specificity in the Insect Immune System: Current Perspectives and Future Challenges. Front. Immunol. 2017, 8, 539. [CrossRef]

27. Coustau, C.; Kurtz, J.; Moret, Y. A Novel Mechanism of Immune Memory Unveiled at the Invertebrate-Parasite Interface. Trends Parasitol. 2016, 32, 353-355. [CrossRef]

28. Melillo, D.; Marino, R.; Italiani, P.; Boraschi, D. Innate Immune Memory in Invertebrate Metazoans: A Critical Appraisal. Front. Immunol. 2018, 9, 1915. [CrossRef]

29. Milutinovic, B.; Kurtz, J. Immune memory in invertebrates. Semin. Immunol. 2016, 28, 328-342. [CrossRef]

30. Pradeu, T.; Du Pasquier, L. Immunological memory: What's in a name? Immunol. Rev. 2018, 283, 7-20. [CrossRef]

31. Arts, R.J.W.; Carvalho, A.; La Rocca, C.; Palma, C.; Rodrigues, F.; Silvestre, R.; Kleinnijenhuis, J.; Lachmandas, E.; Goncalves, L.G.; Belinha, A.; et al. Immunometabolic Pathways in BCG-Induced Trained Immunity. Cell Rep. 2016, 17, 2562-2571. [CrossRef]

32. Arts, R.J.W.; Moorlag, S.; Novakovic, B.; Li, Y.; Wang, S.Y.; Oosting, M.; Kumar, V.; Xavier, R.J.; Wijmenga, C.; Joosten, L.A.B.; et al. BCG Vaccination Protects against Experimental Viral Infection in Humans through the Induction of Cytokines Associated with Trained Immunity. Cell Host Microbe 2018, 23, 89-100.e5. [CrossRef] [PubMed]

33. Kaufmann, E.; Sanz, J.; Dunn, J.L.; Khan, N.; Mendonca, L.E.; Pacis, A.; Tzelepis, F.; Pernet, E.; Dumaine, A.; Grenier, J.C.; et al. BCG Educates Hematopoietic Stem Cells to Generate Protective Innate Immunity against Tuberculosis. Cell 2018, 172, 176-190.e19. [CrossRef] [PubMed]

34. Mukherjee, S.; Subramaniam, R.; Chen, H.; Smith, A.; Keshava, S.; Shams, H. Boosting efferocytosis in alveolar space using BCG vaccine to protect host against influenza pneumonia. PLoS ONE 2017, 12, e0180143. [CrossRef]

35. Ifrim, D.C.; Quintin, J.; Joosten, L.A.; Jacobs, C.; Jansen, T.; Jacobs, L.; Gow, N.A.; Williams, D.L.; van der Meer, J.W.; Netea, M.G Trained immunity or tolerance: Opposing functional programs induced in human monocytes after engagement of various pattern recognition receptors. Clin. Vaccine Immunol. 2014, 21, 534-545. [CrossRef]

36. Divangahi, M.; Aaby, P.; Khader, S.A.; Barreiro, L.B.; Bekkering, S.; Chavakis, T.; van Crevel, R.; Curtis, N.; DiNardo, A.R.; Dominguez-Andres, J.; et al. Trained immunity, tolerance, priming and differentiation: Distinct immunological processes. Nat. Immunol. 2021, 22, 2-6. [CrossRef]

37. Aguilo, N.; Uranga, S.; Marinova, D.; Monzon, M.; Badiola, J.; Martin, C. MTBVAC vaccine is safe, immunogenic and confers protective efficacy against Mycobacterium tuberculosis in newborn mice. Tuberculosis 2016, 96, 71-74. [CrossRef] [PubMed] 
38. White, A.D.; Sibley, L.; Sarfas, C.; Morrison, A.; Gullick, J.; Clark, S.; Gleeson, F.; McIntyre, A.; Arlehamn, C.L.; Sette, A.; et al. MTBVAC vaccination protects rhesus macaques against aerosol challenge with $\mathrm{M}$. tuberculosis and induces immune signatures analogous to those observed in clinical studies. NPJ Vaccines 2021, 6, 4. [CrossRef] [PubMed]

39. Broset, E.; Pardo-Seco, J.; Kanno, A.I.; Aguilo, N.; Dacosta, A.I.; Rivero-Calle, I.; Gonzalo-Asensio, J.; Locht, C.; Leite, L.C.C.; Martin, C.; et al. BCG vaccination improves DTaP immune responses in mice and is associated with lower pertussis incidence in ecological epidemiological studies. EBioMedicine 2021, 65, 103254. [CrossRef]

40. Vierboom, M.P.M.; Dijkman, K.; Sombroek, C.C.; Hofman, S.O.; Boot, C.; Vervenne, R.A.W.; Haanstra, K.G.; van der Sande, M.; van Emst, L.; Dominguez-Andres, J.; et al. Stronger induction of trained immunity by mucosal BCG or MTBVAC vaccination compared to standard intradermal vaccination. Cell Rep. Med. 2021, 2, 100185. [CrossRef]

41. Brooks, N.A.; Puri, A.; Garg, S.; Nag, S.; Corbo, J.; Turabi, A.E.; Kaka, N.; Zemmel, R.W.; Hegarty, P.K.; Kamat, A.M. The association of Coronavirus Disease-19 mortality and prior bacille Calmette-Guerin vaccination: A robust ecological analysis using unsupervised machine learning. Sci. Rep. 2021, 11, 774. [CrossRef]

42. Levi, M.; Miglietta, A.; Romeo, G.; Bartolacci, S.; Ariani, F.; Cipriani, F.; De Filippo, C.; Cavalieri, D.; Balzi, D. Letter in response to article in journal of infection: Impact of routine infant BCG vaccination on COVID-19. J Infect. 2021, 82, e41-e43. [CrossRef] [PubMed]

43. Rivas, M.N.; Ebinger, J.E.; Wu, M.; Sun, N.; Braun, J.; Sobhani, K.; Van Eyk, J.E.; Cheng, S.; Arditi, M. BCG vaccination history associates with decreased SARS-CoV-2 seroprevalence across a diverse cohort of health care workers. J. Clin. Investig. 2021, 131, e145157. [CrossRef] [PubMed] 